\title{
Surgical Treatment of Adult Degenerative Scoliosis
}

\author{
Kyu-Jung Cho ${ }^{1}$, Young-Tae Kim ${ }^{1}$, Sang-hyun Shin ${ }^{1}$, Se-Il Suk ${ }^{2}$ \\ ${ }^{1}$ Department of Orthopedics, Inha University Hospital, Inha University School of Medicine, Incehon, Korea \\ ${ }^{2}$ Seoul Spine Institute, Inje University Sanggye Paik Hospital, Inje University College of Medicine, Seoul, Korea
}

\begin{abstract}
The rapid increase of elderly population has resulted in increased prevalence of adult scoliosis. Adult scoliosis is divided into adult idiopathic scoliosis and adult degenerative scoliosis. These two types of scoliosis vary in patient age, curve pattern and clinical symptoms, which necessitate different surgical indications and options. Back pain and deformity are major indications for surgery in adult idiopathic scoliosis, whereas radiating pain to the legs due to foraminal stenosis is what often requires surgery in adult degenerative scoliosis. When selecting a surgical method, major symptoms and underlying medical diseases should be carefully evaluated, not only to relieve symptoms but also to minimize postoperative complications. Surgical options for adult degenerative scoliosis include: decompression alone; decompression and limited short fusion; and decompression coupled with long fusion and correction of deformity. Decompression and limited short fusion can be applied to patients with a small Cobb's angle and normal sagittal imbalance. For those with a large Cobb's angle and positive sagittal imbalance, long fusion with correction of deformity is required. When long fusion is applied, a careful decision regarding the extent of fusion level should be made when selecting L 5 or S1 as the distal fusion level and T10 or the thoracolumbar junction as the proximal fusion level. For the fusion extending to the sacrum, restoration of sagittal balance and rigid fixation with additional iliac screws should be considered. Any surgical procedures for adult degenerative scoliosis are known to have relatively high occurrences of complications; therefore, risks and benefits should be meticulously considered before selecting a surgical procedure.
\end{abstract}

Keywords: Osteoarthritis spine; Scoliosis; Lumbar vertebrae; Instrumentation; Postoperative complications

\section{Introduction}

Adult scoliosis is defined as spinal deformity with a scoliotic angle of over 10 degrees in skeletally mature patients. The prevalence of adult scoliosis ranges widely from $8.3 \%$ to $68 \%$ of population [1-3], with a higher prevalence occurring among older patients.

While the main symptom of adolescent scoliosis is deformity, the symptoms of adult scoliosis are diverse $[4,5]$. The main symptoms of adult scoliosis are back pain and radiating pain to the legs as well as deformity. Adult sco- liosis is more often accompanied by sagittal and coronal imbalance than adolescent scoliosis. Due to the heterogeneous conditions, conservative treatment does not effectively improve the diverse symptoms. Moreover medical co-morbidity such as cardiopulmonary disease may increase perioperative complications, and osteoporosis may result in a lack of firm fixation.

\section{Classification of Adult Scoliosis}

There are two types of adult scoliosis: adult idiopathic

Received Nov 19, 2013; Revised Dec 20, 2013; Accepted Dec 20, 2013

Corresponding author: Kyu-Jung Cho

Department of Orthopedics, Inha University Hospital, Inha University School of Medicine,

27 Inhang-ro, Jung-gu, Incheon 400-711, Korea

Tel: +32-890-3661, Fax: +32-890-3047, E-mail: chokj@inha.ac.kr 
Table 1. Comparison of adult idiopathic scoliosis with adult degenerative scoliosis

\begin{tabular}{lcc} 
& Adult idiopathic scoliosis & Adult degenerative scoliosis \\
\hline Age & Younger & Older \\
Spinal stenosis & Rare & Common \\
\hline Scoliosis Cobb angle & Large & Small \\
Compensatory thoracic curve & Common & Rare \\
Rotatatory deformity & At entire curve & At apex of curve \\
Lateral subluxation of vertebral body & Not common & Common \\
\hline Coronal and sagittal imbalance & Rare & Common \\
\hline
\end{tabular}

scoliosis, which begins during the adolescent period and continues throughout adulthood; and adult degenerative scoliosis, which develops post-adulthood. Adult degenerative scoliosis usually begins around age fifty $[4,6,7]$. There are several terms used to describe the two types of scoliosis. The former is specifically referred to as adult idiopathic scoliosis or progressive idiopathic scoliosis, while the latter is termed adult degenerative scoliosis, degenerative lumbar scoliosis, de novo scoliosis and primary degenerative scoliosis.

It is difficult to make a clear differentiation between the two types of scoliosis. If a patient's scoliosis begins in adolescence, it is categorized as adult idiopathic scoliosis. However if the patient does not know exactly when the deformity began, the type may not be definitively determined.

The differences between the two types of scoliosis are summarized in Table 1. The average age of patients is higher in adult degenerative scoliosis than in idiopathic scoliosis. Degenerative changes in the vertebral body and discs are more obvious in adult degenerative scoliosis, commonly resulting in spinal stenosis. The Cobb angle is generally below $40^{\circ}$ in adult degenerative scoliosis, while it is much higher in adult idiopathic scoliosis. Indeed Grubb et al. [5] reported that the average angle in adult degenerative scoliosis was $28^{\circ}$, while it was $52^{\circ}$ in idiopathic scoliosis. Adult idiopathic scoliosis has a major curve as well as compensatory curve similar in size to the major curve, while degenerative scoliosis is characterized by a curve in the lumbar area and no compensatory curve in the thoracic vertebra. Rotatory deformity is observed in the entire lumbar spine in adult idiopathic scoliosis, whereas it is limited at the apex of the curve in adult degenerative scoliosis, accompanied with lateral subluxation. Finally sagittal imbalance is more common in degenerative scoliosis (Fig. 1).

\section{Aebi classification}

Aebi [4] classified adult scoliosis into three major types based on the cause of deformity. Type I is primary degenerative scoliosis caused by degenerative changes in the vertebral disc asymmetry and the posterior articulation. Type II is progressive idiopathic scoliosis, which is caused by further development of idiopathic scoliosis that started before adulthood. Type III is secondary adult scoliosis. Type IIIa is caused by extra vertebral causes such as static scoliosis or pelvic inclination. Type IIIb is a type of bone metabolic disease similar to osteoporotic fracture and scoliotic deformity that is caused by weakness of vertebral bone. Aebi classification divides types based on causes, and helps in planning overall treatment and predicting the natural progress of scoliosis. However this method does not aid in deciding on the appropriate operation method, as it does not reflect the specific features and size of the deformity.

\section{Scoliosis Research Society classification}

The Scoliosis Research Society (SRS) introduced a classification system of adult spinal deformity in 2006 [8]. This system categorizes the form of curvature into six different types and three modifiers, referencing the model of King's classification and Lenke classification for adolescent idiopathic scoliosis. The system focuses on radiographic features of spinal deformity and enables comprehensive categorization with inclusion of not only scoliosis, but also kyphosis. Nevertheless this method does not aid in the selection of an operation method or predicting surgical results, as the symptoms and age of patients are not 
considered. The system also does not categorize adult degenerative scoliosis in detail.

\section{Schwab classification}

Schwab classification [9] focuses on the relationship between radiological findings and clinical evaluation, which categorizes the apex of the curve, lumbar lordosis and vertebral body subluxation based on radiological findings. It is found that the lower apex of the curve combined with loss of lordosis lead to poor health-related quality of life (HRQOL). Surgical management is more commonly performed in patients with decreasing lumbar lordosis and higher vertebral subluxation.
A limitation of this system is that it does not describe global balance although the sagittal balance is a significant factor in determining the clinical outcome of adult spinal deformity patients. He revised his classification to include global balance in the sagittal plane [10]. However the revised classification does not consider spino-pelvic parameters that influence sagittal balance.

\section{SRS-Schwab classification}

SRS-Schwab classification was published in 2012 considering the relationship between spino-pelvic parameters and sagittal balance [11]. This system consists of four components: curve type, pelvic incidence minus lumbar
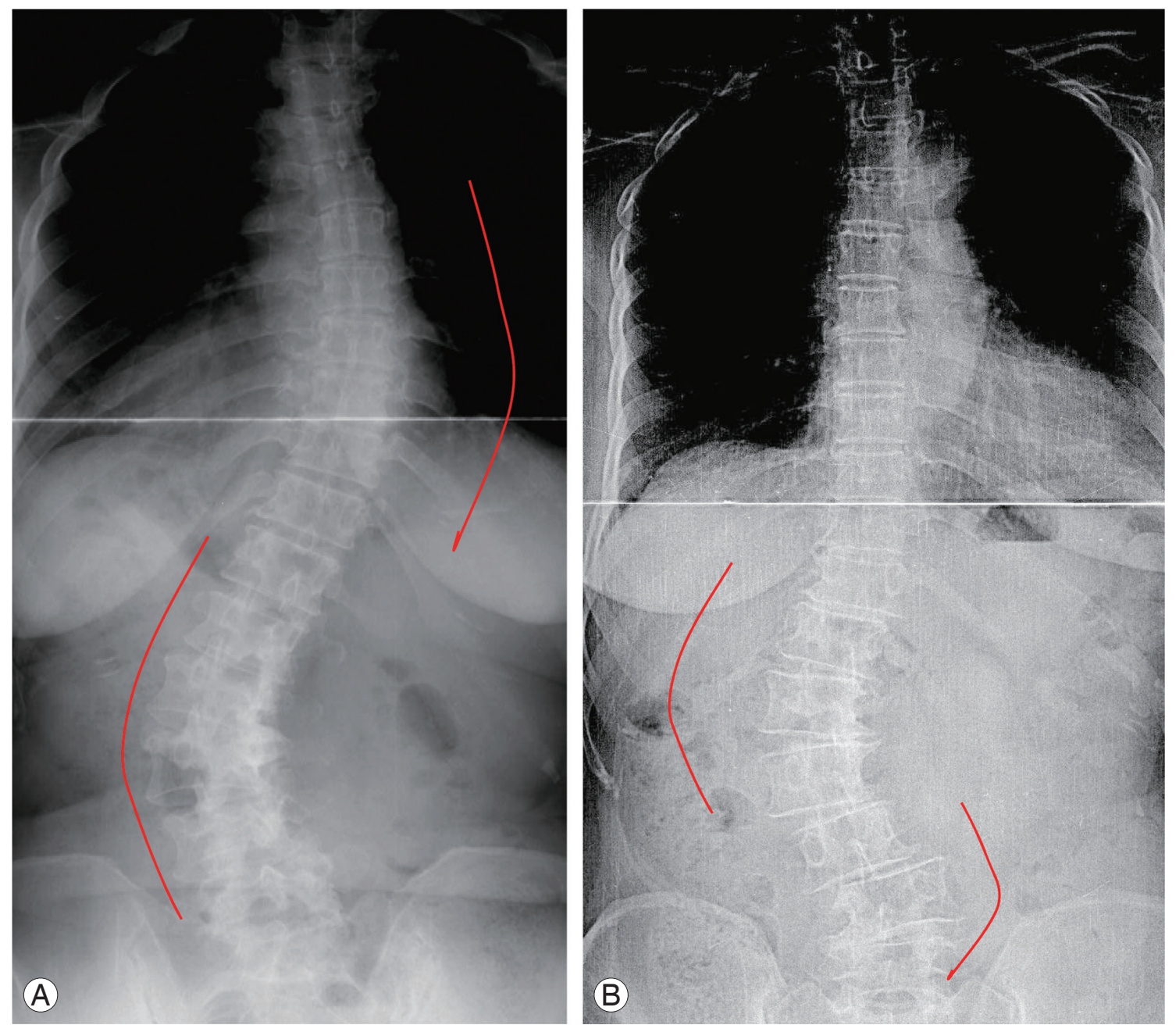

Fig. 1. Radiologic comparison of adult idiopathic scoliosis (A) and adult degenerative scoliosis (B). Both lumbar curve and compensatory thoracic curve exist in similar sizes in adult idiopathic scoliosis (A). Adult degenerative scoliosis (B) generally presents lumbar curve only, without compensatory thoracic curve. Compensatory lumbar curve is seen in the lower lumbar spine. Rotatory deformity is observed in the entire lumbar spine in adult idiopathic scoliosis, whereas it is limited at the apex of the curve in adult degenerative scoliosis, accompanied with lateral subluxation. 
lordosis modifier, global alignment modifier, and pelvic tilt modifier. Curve type is divided into T: thoracic only, L: TL/lumbar only, D: double curve with $\mathrm{T}$ and TL/L curves $>30^{\circ}, \mathrm{N}$ : No major coronal deformity. The study found that HRQOL was closely related to curve type and three sagittal modifiers. Patients with lumbar curve and primary sagittal deformity showed greater disability and poorer health status than patients with thoracic curve or double curve type. Patients with a higher grade of the sagittal modifiers are more likely to have spinal osteotomy surgery, iliac fixation, or decompression surgery. This classification reflects the severity of disease and suggests guideline for treatment. But still there is no suggestion of specific treatment like fusion level of the deformity, so surgical methods should be individualized.

\section{Pathophysiology and Natural History}

The pathophysiology of adult degenerative scoliosis is associated with degenerative change. It is generally accepted that the asymmetrical degeneration of disc and facet joints puts an asymmetric load on the spine that leads to degenerative scoliosis. Another theory is that osteoporosis after menopause leads to degenerative scoliosis, as it is most prevalent in females over the age of fifty [4].

Degenerative scoliosis usually starts after age fifty, and its prevalence and curves increase with age. The risk factors of curve progression may be a curve size over 30 degrees, an asymmetric disc above and below the apical vertebra, lateral subluxation of the apical vertebra over $6 \mathrm{~mm}$ and L5 vertebra being located above rather than below the intercrestal line [12].

\section{Clinical Presentation}

\section{Back pain}

The most common symptom of adult scoliosis is back pain, which usually presents on the convex side of the curvature. The prevalence of back pain varies, but is generally between $60 \%$ and $80 \%$ in adult scoliosis $[6,13]$. Back pain results from degenerative changes in the lumbar disc and facet joint. Muscle fatigue induced by spinal imbalance also causes back pain. The pain is not directly related to the size of curvature; however rotational subluxation and sagittal imbalance may exacerbate back pain $[14,15]$.

\section{Radiating pain and neurological symptoms}

Leg pain presents as intermittent claudication or radiating pain caused by spinal stenosis. Spinal stenosis is present in only $31 \%$ of adult idiopathic scoliosis, whereas it is observed in $90 \%$ of adult degenerative scoliosis [13]. Spinal stenosis is more frequently present as foraminal stenosis than central stenosis, and more common on the concave side than on the convex side. Facet joint hypertrophy and lateral subluxation are associated with foraminal stenosis [16]. Pedicular kinking (kinking of the nerve root between the pedicle and disc) can cause radiating pain on the concave side. When the radiating pain appear on the convex side of the mid lumbar curve, compression of nerve root at L45 and L5S1 on the same side should be meticulously evaluated (Fig. 2). It is common that compensatory curve is formed at the lower lumbar segment (Fig. 1B), in which nerve root is compressed at the concave side. On the convex side, neurological symptoms may also develop by overstretching of the nerve root.

\section{Deformity}

Whereas deformity is the primary symptom in adolescent scoliosis, low back pain or neurological symptoms are chief complaints in adult degenerative scoliosis. In addition to scoliosis, sagittal and coronal imbalance is frequently observed in adult degenerative scoliosis, which causes improper posture leading to low back pain [15].

\section{Surgical Treatment}

When surgical treatment is selected for adult degenerative scoliosis, several points should be considered.

1) General condition including medical co-morbidities: Elderly patients usually have medical co-morbidities so that the incidence of perioperative complications such as cardiopulmonary insufficiency, deep vein thrombosis and infection is quite high.

2) Osteoporosis: Osteoporosis can weaken fixation strength, causing loss of correction and pseudarthrosis. Segmental fixation and anterior column support may strengthen the fixation, and use of cement around the pedicle screw can enhance purchase of screws. In this procedure, bone cement is first injected into the vertebral body through the pedicle, after which pedicle screws are inserted over the cement. 
3) Stiffness of curve: In stiff curve it is difficult to achieve optimal correction with surgery. In adolescent scoliosis, the compensatory curve is spontaneously corrected when the major curve is surgically corrected. However spontaneous correction is not as promising in adult scoliosis due to the decreased flexibility of the curve associated with degenerative change.

4) Coronal and sagittal imbalance: Accompanied coronal and sagittal imbalance is common in degenerative scoliosis. Sagittal imbalance leads to poor results in surgery, so that the restoration of imbalance is more critical than correction of scoliosis itself [15]. Since there are cases of worsening imbalance after surgery, careful attention should be paid to this factor when selecting for the appropriate surgical option $[17,18]$.

The primary indication of surgery in adult degenerative scoliosis is radiating pain to the legs and intermittent claudication. Only for relieving low back pain surgery is rarely indicated. The major cause of radiating pain in degenerative scoliosis is foraminal stenosis, not central stenosis. Disc space narrowing at the concave-side compresses nerve roots, as the vertebral body subluxates laterally. Decompression alone may induce curve progression and worsening of subluxation. Recovery of disc space and correction of lateral subluxation are conducted to relieve radiating pain. Fusion may improve low back pain associated with degenerative changes. Restoration of imbalance relieves the pain caused by muscle fatigue.
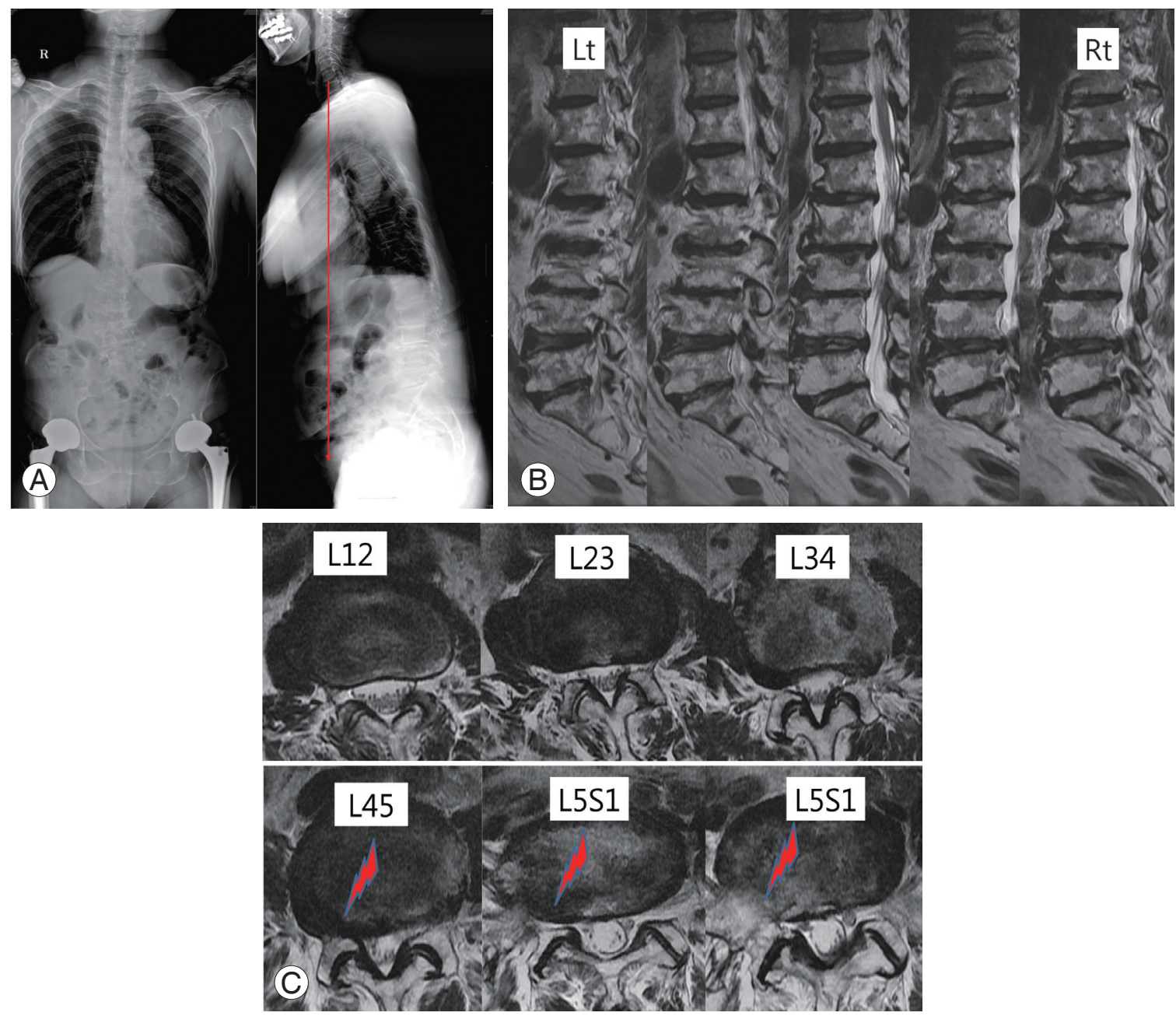

Fig. 2. Radiograph and magnetic resonance imaging (MRI) showing foraminal stenosis due to adult degenerative scoliosis. This 73-year-old woman had complained of low back pain and right leg radiating pain. On whole spine radiograph (A) showed adult lumbar degenerative scoliosis with apex in the right side and sagittal imbalance. Upon sagittal view of MRI (B), spinal stenosis is observed throughout the entire lumbar spine, while foraminal stenosis is more prominent in the right side of L45 and L51 than central stenosis on the axial view (arrows) (C). Foraminal stenosis is often present in the concave side of lower lumbar curve. 
The goal of surgery is to relieve back pain, improve radiating pain and claudication and correct deformity $[19,20]$. A combination of surgical options may be carried out to achieve these goals, including decompression, fusion and/or correction of deformity. Long level fusion including deformity correction may induce excessive blood loss and prolonged surgery time, both of which lead to more postoperative complications. If such complications are anticipated, limited surgery can be selected considering the patient's age and general medical condition. However pain usually recurs when limited surgery is selected, and degenerative change may progress in the non-fused area, eventually causing adjacent segment disease.

Surgical options include: decompression alone; decompression and limited short fusion; and decompression and long fusion with correction of deformity. Surgery should be selected with a full understanding of the cause of symptoms while considering the advantages, disadvantages, indications and complications of each surgical option [21].

\section{Decompression alone}

Although most patients require decompression surgery for radiating pain, decompression alone is not usually recommended in adult degenerative scoliosis. After wide laminectomy and facet joint resection, deformity and instability may worsen, causing recurrence of spinal stenosis [22]. However this method can be applied in elderly patients with poor medical conditions who have a high likelihood of perioperative complications. Nevertheless decompression alone at the apex of curvature is not indicated at which the lateral subluxation is severe. This procedure can be considered in small scoliosis curve without lateral subluxation.

\section{Decompression and limited short fusion (Fig. 3)}

Limited short fusion in which decompression is performed is another option to prevent the spinal instability that arises from decompression alone. Limited short fusion does not involve fusion of the whole curve, but only a decompressed area. This technique is a good choice in moderate scoliosis curve and mild subluxation of the apical vertebra. Adjacent segment disease is a common complication with this method [23]. Degenerative changes may be accelerated outside of fusion when fusion stops within the deformity. Thus fusion should not be stopped at the apex of curvature, but should continue above the apex or stop below the apex.
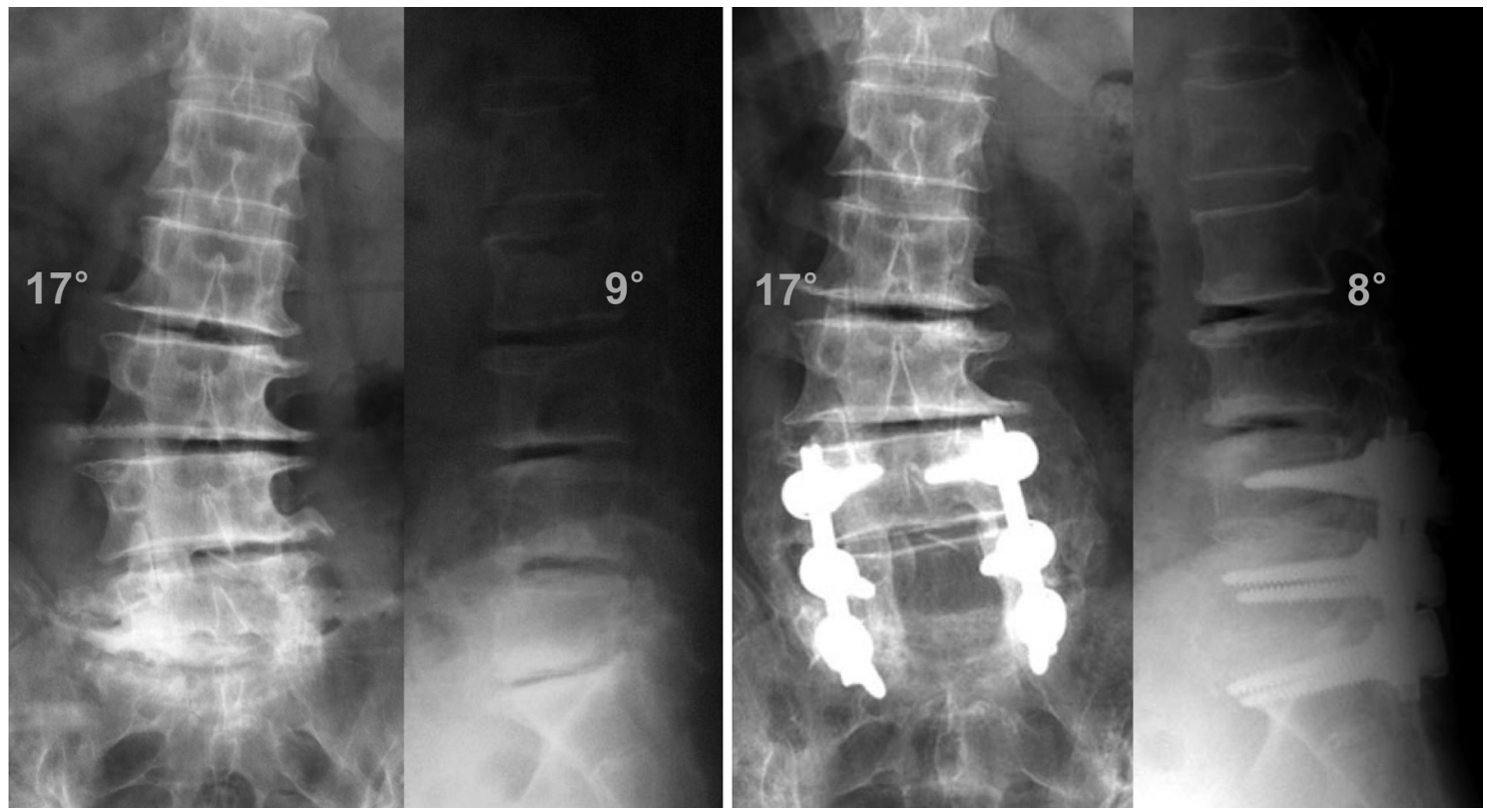

Fig. 3. Decompression and short fusion. Fusion was performed only at L4-5 and L5-S1, where decompression was carried out. Preoperative scoliotic angle of $17^{\circ}$ was not corrected postoperatively at all, because scoliotic deformity was not operated. The problem of short fusion is adjacent segment disease, which is caused by accelerated degenerative change where fusion is not performed. 


\section{Decompression and long fusion with correction of deformity (Fig. 4)}

When the lumbar scoliosis curve is large and subluxation of the apical vertebra is severe, correction of deformity is required. Improvement of back pain and successful fusion are attributed to the correction of scoliosis as well as restoration of lumbar lordosis and sagittal imbalance. Posterior instrumentation can achieve a correction of scoliosis. However it is difficult to restore lumbar lordosis $[6,23]$, which requires anterior interbody release combined with anterior column support. Restoration of sagittal imbalance is achieved by anterior column support or additional techniques such as vertebral osteotomy [24].

\section{Selection of fusion level for correction of deformity}

The fusion level for correction of deformity in degenerative scoliosis has a crucial consideration on the results of surgery. Generally the recognizable criteria to determine fusion level are as follows $[4,7]$ :

1) Fusion should not be stopped at the apex of the curve.

2) The junctional kyphosis is included in the fusion.

3) The severe lateral subluxation is included in the fusion.

4) The spondylolisthesis and retrolisthesis are included in the fusion.

5) The upper instrumented vertebra is better to be horizontal than tilted.

It is debatable whether the proximal fusion level should be extended to T10 or stop at the lumbar spine. Fusion stopped at L1 is likely to cause adjacent segment disease proximally at the thoracolumbar region. To prevent this, fusion up to T10 is recommended since T10 is more stable than T11 and T12 due to true rib attachment on T10. However some surgeons argue that this cannot prevent adjacent segment disease fundamentally, because it develops as a degenerative process [25]. Conversely fusion up to T10 is likely to cause perioperative complications due to the extended length of fusion and long operation time. Cho et al. [26] reported that fusion to T11 or T12 was acceptable when the upper instrumented vertebra was above the upper end vertebra.

It is important to determine whether distal fusion level should be stopped at L5 or extend to the sacrum. The distal fusion usually goes to L5, since the apex of scoliosis is located at L2 -4 and the L4-5 disc has degenerative changes. There is no doubt that fusion to the sacrum is performed in patients who have existing pathology at the L5-S1, such as spinal stenosis, spondylolisthesis and severe degenerative change. However there is controversy regarding whether fusion stops at L5 or extends to the sacrum when the L5-S1 segment looks healthy $[27,28]$.

Surgery in which the fusion stops at L5 compared to S1 is considered to be relatively small; however this may cause subsequent degeneration at L5-S1. Edwards et al. [29] reported that $61 \%$ of patients under fixation at L5 showed degenerative changes, leading to sagittal imbalance and increasing risk of reoperation. Accordingly it is preferable to fuse to the $\mathrm{S} 1$ in patients with sagittal imbalance, as it is highly likely to cause subsequent degeneration at the L5-S1 segment, even without degenerative change before surgery.

Fusion to the sacrum achieve a better correction of sagittal imbalance than fusion to L5. However the complication rate is higher in the fusion to the sacrum. Pseudarthrosis, which is the most common complication at the L5-S1 segment, developed in $42 \%$ of cases of fusion to the sacrum, but $4 \%$ of cases of fusion to L5 [29]. To prevent pseudarthrosis, interbody fusion and additional iliac fixation are strongly recommended [30]. Sagittal decompensation after fusion to the sacrum is not uncommon, and therefore restoration of lumbar lordosis is critical to achieve sagittal balance [18].

\section{Postoperative Complications}

Excessive blood loss is not uncommon in the surgery of adult deformity, which is closely related to perioperative complications such as epidural hematoma, pulmonary embolism and respiratory failure. Increased fusion level and longer operation time are associated with more blood loss [31]. Additionally low-dose aspirin is gaining popularity owing to its potential to prevent cardiovascular disease, which makes the bleeding-control difficult. Although aspirin is not taken for seven days before surgery, blood drainage is still higher in patients that regularly take aspirin than in those who do not. Minimizing blood loss is needed to reduce perioperative complications, and preoperative autologous blood donation, antifibrinolytic administration and blood salvage can be attempted to achieve this goal.

Minimally invasive techniques have been introduced for adult degenerative scoliosis to reduce periopera- 


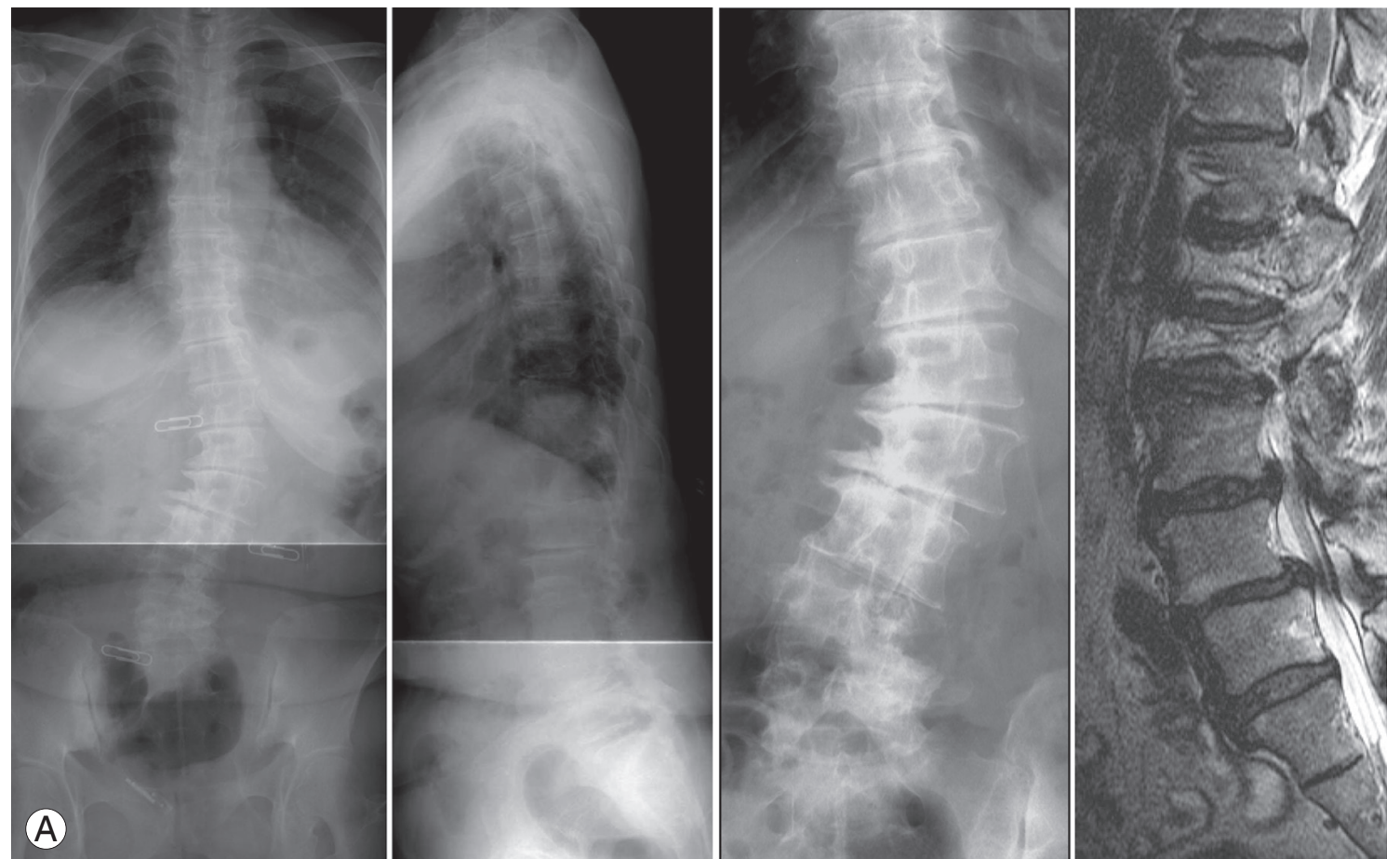

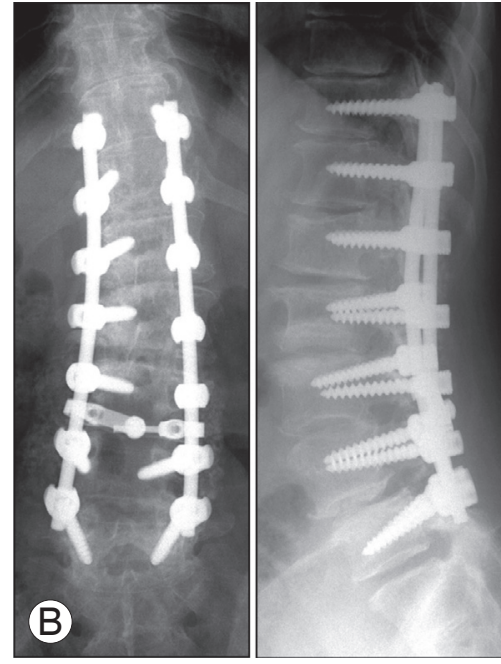

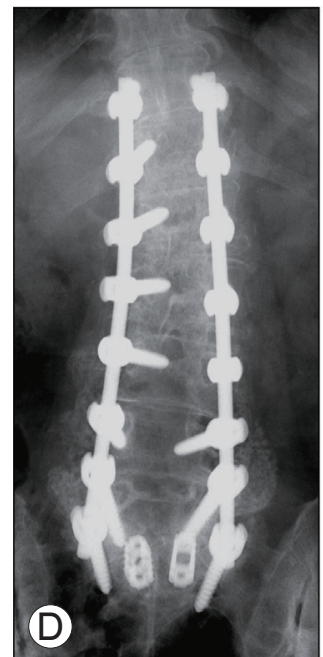

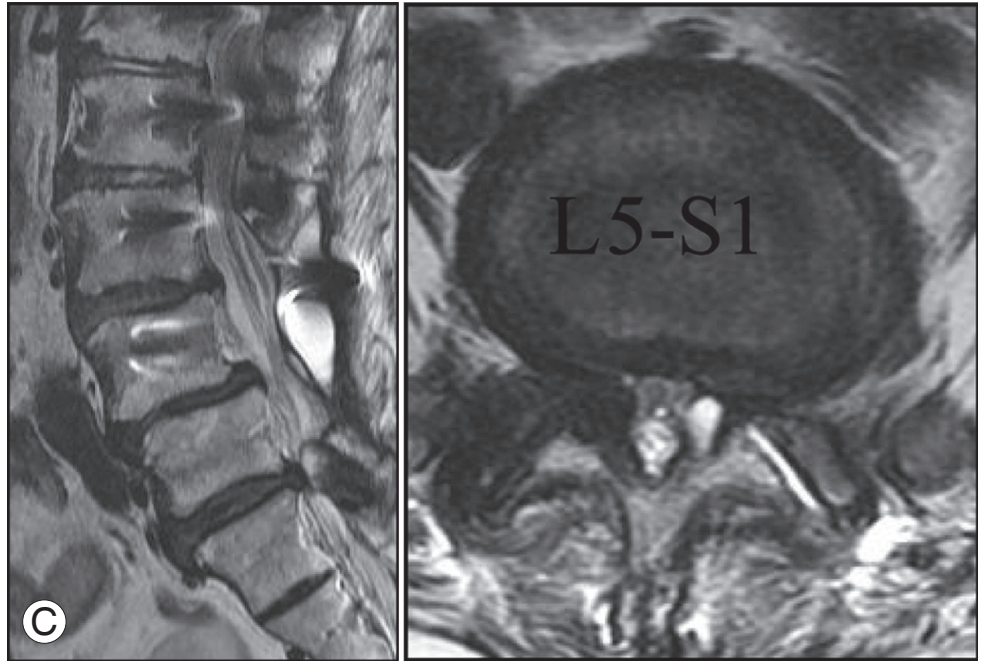

Fig. 4. Long fusion and fixation to the sacrum with correction of deformity. (A) This 72-yearold woman had adult degenerative scoliosis with $35^{\circ}$ Cobb angle scoliosis and multi-level spinal stenosis. (B) Decompression and fusion at T11$\mathrm{L} 5$ was performed as the L5-S1 disc was healthy on magnetic resonance imaging. (C) One year after surgery, spinal stenosis developed at L5-S1. (D) A revision surgery (decompression and extension of fusion to the sacrum) was performed Subsequent disc degeneration at L5-S1, as often present when fusion is stopped at L5. Complications such as pseudarthrosis or implant failure are developed at the lumbosacral junction after long fusion. 
tive complications. Lateral lumbar interbody fusion is performed with less blood loss and less morbidity than open correction. However some limitations of the lateral interbody fusion technique have been reported $[32,33]$. Surgery at the L5-S1 segment cannot be conducted via the transpsoas approach, because the iliac crest hinders the approach. The approach-related complications such as sensory deficits in the thigh area are not completely prevented with intraoperative neuromonitoring.

Late complications include adjacent segment disease, pseudarthrosis and instrumentation failure. Adjacent segment disease presents with spinal stenosis and proximal junctional kyphosis $[18,34]$. The risk factors of adjacent segment disease appear to be sagittal imbalance, facet joint injury and elderly patients.

Pseudarthrosis most commonly occurs at L5-S1 when fused to the sacrum [35], but it also occurs at the thoracolumbar junction. The incidence of pseudarthrosis is high when the patient is older, thoracolumbar kyphosis is more than $20^{\circ}$ and the lumbar lordosis is decreased. Sagittal imbalance is another risk factor of pseudarthrosis.

Instrumentation failure results from screw loosening and pullout at the most distal part and the superior part of fixation [36]. Screw loosening occurs in patients with sagittal imbalance, especially in long level fusion. Cement augmentation on the proximal screws can help reduce screw loosening. To prevent failure of fixation in the distal part of instrumentation, firm fixation with additional iliac screws is suggested. Because L5 screw loosening is common in patients with sagittal imbalance, it is recommended that this fusion be extended to the sacrum $[37,38]$.

Adult degenerative scoliosis is often accompanied by sagittal imbalance. Although restoration of sagittal imbalance is critical in order to improve clinical outcome, failure of sagittal realignment after instrumentation was observed in some patients $[18,39,40]$. Higher pelvic incidence and larger pelvic tilt may be associated with sagittal decompensation due to insufficient correction. In those patients a higher degree of correction of lumbar lordosis is needed. The amount of correction of lumbar lordosis should be proportional to the measured pelvic incidence. Loss of correction in disc spaces after instrumentation may also cause suboptimal correction of lumbar lordosis. Anterior column support with cages is useful to prevent loss of correction in disc spaces [39].

\section{Conclusions}

Patients with adult degenerative scoliosis have various symptoms, including back pain, leg pain, claudication, scoliosis and coronal and sagittal imbalance. The heterogeneous conditions may lend managing patients difficult. There are three surgical options for adult degenerative scoliosis: decompression alone; decompression and limited short fusion; and long fusion with correction of deformity. Decompression alone is not usually recommended due to curve progression after surgery. Limited short fusion is performed in decompressed segments. This procedure is indicated in cases with less Cobb angle, minimal rotational deformity and no coronal and sagittal imbalance. With this short fusion, correction of scoliosis and restoration of lumbar lordosis are not adequate; and therefore all segments of deformity should be included in the fusion to correct the deformity. This long fusion is indicated in cases of severe Cobb angle and coronal and sagittal imbalance.

The goal of surgery is not only to relieve pain and correct deformity, but also to minimize complications related to surgery, as the incidence of complications is relatively high. Limited short fusion is likely to cause proximal adjacent segment disease in the un-fused segments. Long fusion with correction of deformity causes more blood loss and high perioperative complications. Accordingly surgeons should attempt to seek a trade-off between the benefits of surgery and risks of complications. One should consider the patient's general condition, not just that of the spine, when choosing surgical options.

\section{Conflict of Interest}

No potential conflict of interest relevant to this article was reported.

\section{References}

1. Carter OD, Haynes SG. Prevalence rates for scoliosis in US adults: results from the first National Health and Nutrition Examination Survey. Int J Epidemiol 1987;16:537-44.

2. Schwab F, Dubey A, Gamez L, et al. Adult scoliosis: prevalence, SF-36, and nutritional parameters in an elderly volunteer population. Spine (Phila Pa 1976) 2005;30:1082-5. 
3. Hong JY, Suh SW, Modi HN, Hur CY, Song HR, Park $\mathrm{JH}$. The prevalence and radiological findings in 1347 elderly patients with scoliosis. J Bone Joint Surg Br 2010;92:980-3.

4. Aebi M. The adult scoliosis. Eur Spine J 2005;14:92548.

5. Grubb SA, Lipscomb HJ, Coonrad RW. Degenerative adult onset scoliosis. Spine (Phila Pa 1976) 1988;13:241-5.

6. Daffner SD, Vaccaro AR. Adult degenerative lumbar scoliosis. Am J Orthop (Belle Mead NJ) 2003;32:7782.

7. Gupta MC. Degenerative scoliosis. Options for surgical management. Orthop Clin North Am 2003;34:269-79.

8. Lowe T, Berven SH, Schwab FJ, Bridwell KH. The SRS classification for adult spinal deformity: building on the King/Moe and Lenke classification systems. Spine (Phila Pa 1976) 2006;31(19 Suppl):S119-25.

9. Schwab F, Farcy JP, Bridwell K, et al. A clinical impact classification of scoliosis in the adult. Spine (Phila Pa 1976) 2006;31:2109-14.

10. Schwab F, Lafage V, Farcy JP, et al. Surgical rates and operative outcome analysis in thoracolumbar and lumbar major adult scoliosis: application of the new adult deformity classification. Spine (Phila Pa 1976) 2007;32:2723-30.

11. Schwab F, Ungar B, Blondel B, et al. Scoliosis Research Society-Schwab adult spinal deformity classification: a validation study. Spine (Phila Pa 1976) 2012;37:1077-82.

12. Pritchett JW, Bortel DT. Degenerative symptomatic lumbar scoliosis. Spine (Phila Pa 1976) 1993;18:7003.

13. Grubb SA, Lipscomb HJ, Suh PB. Results of surgical treatment of painful adult scoliosis. Spine (Phila Pa 1976) 1994;19:1619-27.

14. Weinstein SL, Dolan LA, Cheng JC, Danielsson A, Morcuende JA. Adolescent idiopathic scoliosis. Lancet 2008;371:1527-37.

15. Glassman SD, Bridwell K, Dimar JR, Horton W, Berven S, Schwab F. The impact of positive sagittal balance in adult spinal deformity. Spine (Phila Pa 1976) 2005;30:2024-9.

16. Simmons ED. Surgical treatment of patients with lumbar spinal stenosis with associated scoliosis. Clin Orthop Relat Res 2001;(384):45-53.
17. Schwab FJ, Blondel B, Bess S, et al. Radiographical spinopelvic parameters and disability in the setting of adult spinal deformity: a prospective multicenter analysis. Spine (Phila Pa 1976) 2013;38:E803-12.

18. Cho KJ, Suk SI, Park SR, et al. Risk factors of sagittal decompensation after long posterior instrumentation and fusion for degenerative lumbar scoliosis. Spine (Phila Pa 1976) 2010;35:1595-601.

19. Bradford DS, Tay BK, Hu SS. Adult scoliosis: surgical indications, operative management, complications, and outcomes. Spine (Phila Pa 1976) 1999;24:261729.

20. Marchesi DG, Aebi M. Pedicle fixation devices in the treatment of adult lumbar scoliosis. Spine (Phila Pa 1976) 1992;17(8 Suppl):S304-9.

21. Akbarnia BA, Ogilvie JW, Hammerberg KW. Debate: degenerative scoliosis: to operate or not to operate. Spine (Phila Pa 1976) 2006;31(19 Suppl):S195-201.

22. Vaccaro AR, Ball ST. Indications for instrumentation in degenerative lumbar spinal disorders. Orthopedics 2000;23:260-71.

23. Cho KJ, Suk SI, Park SR, et al. Short fusion versus long fusion for degenerative lumbar scoliosis. Eur Spine J 2008;17:650-6.

24. Bradford DS, Tribus CB. Vertebral column resection for the treatment of rigid coronal decompensation. Spine (Phila Pa 1976) 1997;22:1590-9.

25. Shufflebarger H, Suk SI, Mardjetko S. Debate: determining the upper instrumented vertebra in the management of adult degenerative scoliosis: stopping at T10 versus L1. Spine (Phila Pa 1976) 2006;31(19 Suppl):S185-94.

26. Cho KJ, Suk SI, Park SR, Kim JH, Jung JH. Selection of proximal fusion level for adult degenerative lumbar scoliosis. Eur Spine J 2013;22:394-401.

27. Bridwell KH, Edwards CC 2nd, Lenke LG. The pros and cons to saving the L5-S1 motion segment in a long scoliosis fusion construct. Spine (Phila Pa 1976) 2003;28:S234-42.

28. Polly DW Jr, Hamill CL, Bridwell KH. Debate: to fuse or not to fuse to the sacrum, the fate of the L5-S1 disc. Spine (Phila Pa 1976) 2006;31(19 Suppl): S179-84.

29. Edwards CC 2nd, Bridwell KH, Patel A, et al. Thoracolumbar deformity arthrodesis to L5 in adults: the fate of the L5-S1 disc. Spine (Phila Pa 1976) 2003; 28:2122-31.

30. Cho KJ, Suk SI, Park SR, et al. Arthrodesis to L5 ver- 
sus S1 in long instrumentation and fusion for degenerative lumbar scoliosis. Eur Spine J 2009;18:531-7.

31. Cho KJ, Suk SI, Park SR, et al. Complications in posterior fusion and instrumentation for degenerative lumbar scoliosis. Spine (Phila Pa 1976) 2007;32:22327.

32. Isaacs RE, Hyde J, Goodrich JA, Rodgers WB, Phillips FM. A prospective, nonrandomized, multicenter evaluation of extreme lateral interbody fusion for the treatment of adult degenerative scoliosis: perioperative outcomes and complications. Spine (Phila Pa 1976) 2010;35(26 Suppl):S322-30.

33. Dakwar E, Cardona RF, Smith DA, Uribe JS. Early outcomes and safety of the minimally invasive, lateral retroperitoneal transpsoas approach for adult degenerative scoliosis. Neurosurg Focus 2010;28:E8.

34. Kim YJ, Bridwell KH, Lenke LG, Rhim S, Cheh G. Sagittal thoracic decompensation following long adult lumbar spinal instrumentation and fusion to L5 or S1: causes, prevalence, and risk factor analysis. Spine (Phila Pa 1976) 2006;31:2359-66.

35. Kim YJ, Bridwell KH, Lenke LG, Cho KJ, Edwards CC 2nd, Rinella AS. Pseudarthrosis in adult spinal deformity following multisegmental instrumentation and arthrodesis. J Bone Joint Surg Am 2006;88:721-8. 36. Emami A, Deviren V, Berven S, Smith JA, Hu SS, Bradford DS. Outcome and complications of long fusions to the sacrum in adult spine deformity: luquegalveston, combined iliac and sacral screws, and sacral fixation. Spine (Phila Pa 1976) 2002;27:776-86.

37. Pateder DB, Kebaish KM, Cascio BM, Neubaeur P, Matusz DM, Kostuik JP. Posterior only versus combined anterior and posterior approaches to lumbar scoliosis in adults: a radiographic analysis. Spine (Phila Pa 1976) 2007;32:1551-4.

38. Tsuchiya K, Bridwell KH, Kuklo TR, Lenke LG, Baldus C. Minimum 5-year analysis of L5-S1 fusion using sacropelvic fixation (bilateral S1 and iliac screws) for spinal deformity. Spine (Phila Pa 1976) 2006;31:303-8.

39. Cho KJ, Kim KT, Kim WJ, et al. Pedicle subtraction osteotomy in elderly patients with degenerative sagittal imbalance. Spine (Phila Pa 1976) 2013;38:E1561-6.

40. Schwab F, Patel V, Shaffrey CI, et al. Sagittal realignment failures following pedicle subtraction osteotomy surgery: are we doing enough?: Clinical article. J Neurosurg Spine 2012;16:539-46. 